\title{
Remarks on the Iterative Solution of the Neumann Problem on a Rectangle by Successive Line Over-Relaxation*
}

\author{
By Fred W. Dorr
}

\begin{abstract}
Successive line over-relaxation can be used to solve the equations for certain finite-difference analogs of the Neumann problem for Poisson's equation on a rectangle. In this note, asymptotic estimates for the choice of relaxation parameter and rate of convergence of this method are collected. These results are then applied to some recent computational experiments carried out by John Gary.
\end{abstract}

Introduction. Approximate solutions to the system of equations corresponding to a finite-difference analog of the Neumann problem can be found iteratively by successive line over-relaxation (SLOR) [4]. In a recent paper [3] John Gary carried out a computational study of this problem and the related Dirichlet problem. In particular, when he used SLOR to solve the finite difference equations for Poisson's equation on a rectangle, he noticed a distinct difference in the rates of convergence for Dirichlet and Neumann boundary conditions. This observation can be readily accounted for by using results of Keller [5] and Parter [6]. In this note we collect the pertinent formulae, and use them to explain the behavior of the solutions of the particular problem that Gary considered.

The Neumann Problem. Let $R$ be the rectangle $(0, a) \times(0, b)$, and introduce a mesh on $\bar{R}$ of width $\Delta x$ in the $x$-direction and $\Delta y$ in the $y$-direction. We assume that $\Delta x=a\left(N_{x}+1\right)^{-1}$ and $\Delta y=b\left(N_{y}+1\right)^{-1}$ for integers $N_{x}$ and $N_{y}$. Using the usual 5 -point approximation for $\Delta u$, and the usual first order extrapolation for the normal derivative $\partial u / \partial v$ (see [3]), we write the finite-difference equations as a matrix equation $\& u=\rho$. We assume that the vector $u=\left(u_{i j}\right)$ is ordered in blocks of vertical columns, with $u_{i j}=u(i \Delta x, j \Delta y)$. We can take $\&$ to have the form $\&=D+U+U^{T}$, where $D$ is a block diagonal matrix with positive-definite entries, and $U$ is a block matrix with entries $-\alpha I$ on the super-diagonal, where $\alpha=(\Delta y)^{2}(\Delta x)^{-2}$. The $N_{x} N_{y} \times N_{x} N_{y}$ matrix $\&$ is positive semidefinite with a one-dimensional null-space, which is spanned by the vector e all of whose components are 1 .

For a real parameter $\gamma$ with $1 / 2<\gamma<1$ we define $N=\gamma D+U^{T}$ and $P=$ $(\gamma-1) D-U$. Then $N$ is nonsingular, so we define $M=N^{-1} P$, and the (vertical) SLOR iteration takes the form

$$
u^{(0)} \text { arbitrary, } \quad u^{(n+1)}=M u^{(n)}+N^{-1} \rho .
$$

If we assume that $\rho^{T} \mathbf{e}=0$, so that $\mathfrak{L} u=\rho$ has a solution, then, for any choice of

Received February 19, 1967, revised May 23, 1968.

* Sponsored by the Mathematics Research Center, United States Army, Madison, Wisconsin, under Contract No. DA-31-124-ARO-D-462. 
$u^{(0)}$, the iterates $u^{(n)}$ converge to a solution of $\& u=\rho$ [4, p. 285]. If we define $\epsilon^{(n)}=$ $u-u^{(n)}$, then $\epsilon^{(n)}=M^{n} \epsilon^{(0)}$. An elementary calculation then shows that

$$
\left\|u^{(n)}-u^{*}\right\|_{\infty} \sim c\left(\begin{array}{c}
n \\
p_{0}-1
\end{array}\right) \lambda_{0}{ }^{n+1-p_{0}},
$$

where $\mathcal{L} u^{*}=\rho, c$ is a constant that depends only on the initial guess $u^{(0)}, \lambda_{0}=$ $\max \{|\lambda| \mid \lambda=$ eigenvalue of $M,|\lambda|<1\}$, and $p_{0}$ is the maximum degree of any Jordan block corresponding to an eigenvalue $\lambda$ of $M$ with $|\lambda|=\lambda_{0}$.

The Rate of Convergence. We now define the rate of convergence to be $r_{0}=$ $-\ln \lambda_{0}$. The question of estimating $\lambda_{0}$ can be reduced to the problem of estimating the eigenvalues of the iteration matrix $M_{1}$ for the corresponding Jacobi iterative procedure [2, p. 250]. In particular, by using these well-known techniques, it follows that if we use an optimum relaxation parameter $\gamma_{0}=\frac{1}{2}\left(1+\left(1-\mu_{0}^{2}\right)^{1 / 2}\right)$, then

$$
\lambda_{0}=\left(1-\left(1-\mu_{0}^{2}\right)^{1 / 2}\right)\left(1+\left(1-\mu_{0}^{2}\right)^{1 / 2}\right)^{-1} .
$$

The quantity $\mu_{0}$ is defined by $\mu_{0}=\max \left\{|\mu| \mid \mu=\right.$ eigenvalue of $\left.M_{1},|\mu|<1\right\}$, and an estimate for $\mu_{0}$ is provided by the following theorem of Parter [6, p. 343]:

THeOREM 1. Let $\Lambda$ be the smallest nonzero eigenvalue $\lambda$ of the analytic problem

$$
\begin{aligned}
\Delta u+\lambda u & =0 \quad \text { in } R, \\
\partial u / \partial v & =0 \text { on } \partial R, \\
u & \neq 0 .
\end{aligned}
$$

Then as $\Delta x$ and $\Delta y$ tend to $0, \mu_{0} \sim 1-(\Lambda / 2)(\Delta x)^{2}+o(\Delta x \Delta y)$.

For the Neumann problem on a rectangle, these eigenvalues are [1, p. 429] $\lambda_{m n}=\pi^{2}\left(m^{2} a^{-2}+n^{2} b^{-2}\right)$ for $m, n \geqq 0$. Thus $\Lambda=\pi^{2} c^{-2}$ for $c=\max (a, b)$. This can be summarized in the following

THEOREM 2. If SLOR is used to solve the finite-difference equations for the Neumann problem on a rectangle, with optimal relaxation parameter $\gamma_{0} \sim \frac{1}{2}\left(1+\pi c^{-1} \Delta x\right)$, then $\lambda_{0} \sim\left(1-2 \pi c^{-1} \Delta x\right)$. Thus the asymptotic rate of convergence is $r_{0} \sim 2 \pi c^{-1} \Delta x$.

Application to the Results of Gary. In [3] Gary considered the following class of problems:**

Let $R(\alpha)=(0, a) \times\left(0, \alpha^{1 / 2} a\right)$ for $0<\alpha \leqq 1$. Solve the Dirichlet and Neumann problems on $R(\alpha)$ by SLOR with $N_{x}$ and $N_{y}$ fixed (using the optimal relaxation parameter), and let the rates of convergence be $r_{D}(\alpha)$ and $r_{N}(\alpha)$ respectively.

Gary observed that $r_{D}(\alpha)$ increases as $\alpha$ decreases. This agrees with the known results for the Dirichlet problem [5], and in fact it is easy to verify the asymptotic estimate, $r_{D}(\alpha) \sim 2 \pi\left(N_{x}+1\right)^{-1}\left(1+\alpha^{-1}\right)^{1 / 2}$. In addition, Gary conjectured that $r_{N}(\alpha)$ is independent of $\alpha$. This now follows immediately from Theorem 2 , since $r_{N}(\alpha) \sim 2 \pi\left(N_{x}+1\right)^{-1}$.

** There is an inconsistency in Gary's paper [3]. Namely, if $N_{x}=N_{y}=40, a=4 \pi$ and $b=2 \pi$ then $\alpha=1 / 4$, so that $\alpha$ cannot vary. There are several ways to resolve this difficulty, and Dr. Gary was kind enough to send a copy of his computer program so that we could verify that the problem as stated above is the one he actually solved. 
University of Wisconsin

Madison, Wisconsin 53706

1. R. Courant \& D. Hilbert, Methoden der Mathematischen Physik. Vol. I, Interscience, New York, 1953; Russian transl., GITTL, Moscow, 1951. MR 13, 800; MR 16, 426.

2. G. Forsythe \& W. Wasow, Finite-Difference Methods for Partial Differential Equations, Wiley, New York, 1960; Russian transl., Moscow, 1963. MR 23 \#B3156.

3. J. GARY, "On convergence rates for line overrelaxation," Math. Comp., v. 21, 1967, pp. 220-223.

4. H. B. KeLler, "On the solution of singular and semidefinite linear systems by iteration," J. Soc. Indust. Appl. Math. Ser. B Numer. Anal., v. 2, 1965, pp. 281-290. MR 33 \#3447.

5. H. B. KELLER, "On some iterative methods for solving elliptic difference equations," Quart. Appl. Math., v. 16, 1958, pp. 209-226. MR 22 \#8667.

6. S. V. PARTER, "On estimating the 'rates of convergence' of iterative methods for elliptic difference equations," Trans. Amer. Math. Soc., v. 114, 1965, pp. 320-354. MR 31 \#5350. 